\title{
Guest control of structure in porous organic cages $\dagger$
}

Cite this: Chem. Commun., 2014, 50,9465

Received 30th May 2014, Accepted 25th June 2014

DOI: $10.1039 / \mathrm{c} 4 \mathrm{cc} 04158 \mathrm{e}$

www.rsc.org/chemcomm

\author{
Marc A. Little, Samantha Y. Chong, Marc Schmidtmann, Tom Hasell and \\ Andrew I. Cooper*
}

Two porous organic cages with different thermodynamic polymorphs were induced by co-solvents to interchange their crystal packing modes, thus achieving guest-mediated control over solid-state porosity. In situ crystallography allows the effect of the co-solvent guests on these structural interconversions to be understood.

Porous molecular solids ${ }^{1}$ comprise non-covalent intermolecular interactions that are weaker and often less directional than the coordination or covalent bonds that define zeolites, ${ }^{2}$ metal-organic frameworks (MOFs), ${ }^{3}$ and covalent organic frameworks (COFs). ${ }^{4}$ Structural polymorphism is therefore common, and this raises challenges for the targeted design of solid-state function.

We have developed porous organic cage molecules via [4+6] cycloimination reactions of 1,3,5-triformylbenzene (TFB) with vicinal aliphatic diamines such as $(1 R, 2 R)$-diaminocyclohexane (CC3- $R$ ) and $(1 R, 2 R)$-diaminocyclopentane (CC4- $R$ ) (Fig. 1a). ${ }^{5}$ The molecular solubility of organic cages ${ }^{6}$ allows crystallisation to be decoupled from synthesis, unlike MOFs and COFs where these two processes constitute the same step.

CC3- $R$ prefers to pack in a window-to-window arrangement, 5,7 which generates an interconnected diamondoid pore network (Fig. 1b). This thermodynamic polymorph, $\mathbf{C C 3} \alpha$, has an apparent BET surface area $\left(\mathrm{SA}_{\mathrm{BET}}\right)$ of $\sim 410 \mathrm{~m}^{2} \mathrm{~g}^{-1}$ in the crystalline state. ${ }^{7}$ Both crystal structure predictions $(\mathrm{CSP})^{8}$ and DFT 'cage dimer' calculations $^{7}$ rationalise this crystal packing. By contrast, CC4- $R$ packs via a window-to-arene interaction, despite its close structural similarity with CC3- $R$, and this results in a 2-D pore structure in its thermodynamic polymorph, $\mathbf{C C} 4 \alpha$, rather than a 3-D diamondoid pore structure (Fig. 1c).

In this study, we show that these two thermodynamic 'alpha' crystal packing modes can be interchanged by using specific co-solvents to direct the crystal packing, such that CC3- $R$ packs

Department of Chemistry and Centre for Materials Discovery, University of Liverpool, Crown Street, Liverpool, L69 7ZD, UK. E-mail: aicooper@liv.ac.uk; Web: http://www.liv.ac.uk/cooper-group/

$\dagger$ Electronic supplementary information (ESI) available: Full synthetic, gas sorption and crystallographic details. CCDC 991214-991219. For ESI and crystallographic data in CIF or other electronic format see DOI: 10.1039/c4cc04158e
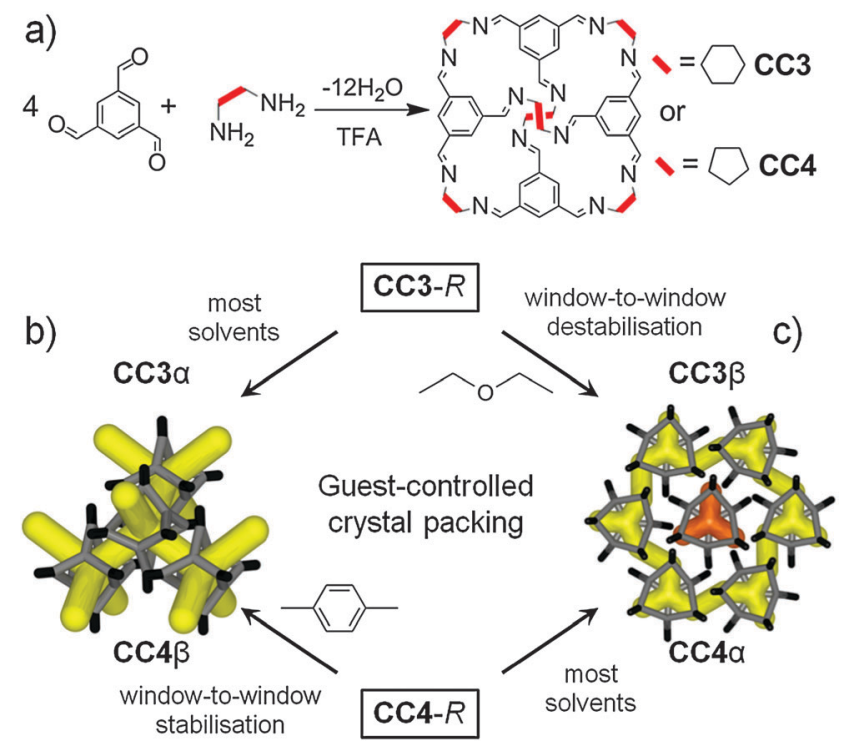

Fig. 1 (a) Synthesis of organic cage molecules CC3 and CC4. Scheme showing interchangeable crystal packings for cages CC3 and CC4, (b) 3-D diamondoid pore channels, and (c) 2-D layered pore structure with formally disconnected voids. Orange $=$ disconnected voids; yellow $=$ interconnected pores.

like CC4- $R$, and vice versa (Fig. $1 \mathrm{~b}$ and $\mathrm{c}$ ). We showed recently that 1,4-dioxane can direct three different tetrahedral cage molecules (CC1, CC2 and CC13) ${ }^{9}$ to crystallise isostructurally with $\mathbf{C C} 3 \alpha$, providing that the cages comprise a racemic mixture.

Here we expand this concept to the homochiral cage molecules CC3- $R$ and CC4- $R$. We direct these two cages into isostructural packings, and hence achieve control over pore topology, pore volume, and surface area. Computational CSP can map the energy landscape for solvated crystal structures ${ }^{11}$ or for solvent-free organic cage crystals, ${ }^{8,10}$ but this is not yet computationally affordable for cage solvates. Our strategy, therefore, was to combine intuitive concepts of shape-direction, ${ }^{9}$ solvent screening, and CSP calculations for solvent-free crystals. ${ }^{10}$

CC3- $R$ is readily soluble in $\mathrm{CH}_{2} \mathrm{Cl}_{2}$ but it is insoluble in $\mathrm{Et}_{2} \mathrm{O}$. However, the addition of excess of an antisolvent, $\mathrm{Et}_{2} \mathrm{O}$, to solutions 

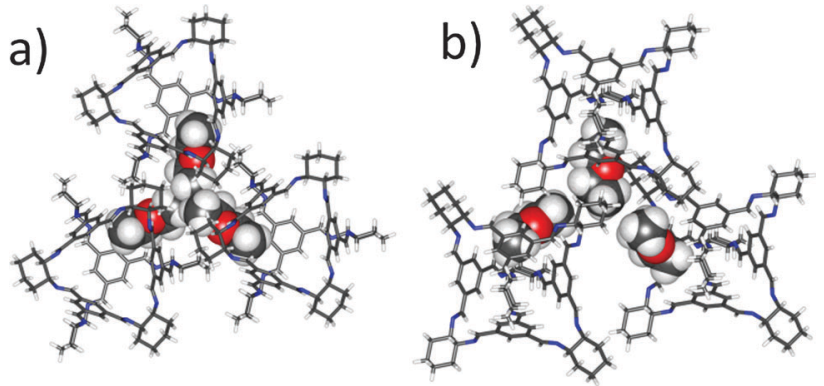

Fig. 2 Single crystal structure for $\mathrm{CC} 3-R \cdot\left(\mathrm{Et}_{2} \mathrm{O}\right)_{3} \cdot \mathrm{CH}_{2} \mathrm{Cl}_{2}$, viewed parallel to [001] axis (a) and off axis (b). $\mathrm{Et}_{2} \mathrm{O}$ solvent molecules are shown in space filling representation, with the $\mathrm{CH}_{2} \mathrm{Cl}_{2}$ molecules in the cage cavities omitted for clarity.

of CC3- $R$ in $\mathrm{CH}_{2} \mathrm{Cl}_{2}$ did not result in direct precipitation of the cage, even when a twentyfold volumetric excess of $\mathrm{Et}_{2} \mathrm{O}$ was added. Instead, slow evaporation of the resultant homogenous solution affords hexagonal, needle-shaped single crystals of $\left(\right.$ CC3-R) $\cdot\left(\mathrm{Et}_{2} \mathrm{O}\right)_{3} \cdot \mathrm{CH}_{2} \mathrm{Cl}_{2}$ (Fig. S1, ESI $\dagger$ ), which crystallise in the trigonal space group $R 3$. The asymmetric unit comprises one third of a CC3- $R$ molecule positioned on a threefold rotation axis, plus one $\mathrm{CH}_{2} \mathrm{Cl}_{2}$ molecule in the intrinsic cage cavity and one well-ordered $\mathrm{Et}_{2} \mathrm{O}$ solvent molecule in the window site (Fig. 2). Overall, $\mathrm{Et}_{2} \mathrm{O}$ occupies three of the four cage window sites, with one hydrophobic methyl terminus directed toward the hydrophobic cage cavity, sharing the cage void with $\mathrm{CH}_{2} \mathrm{Cl}_{2}$.

The inclusion of $\mathrm{Et}_{2} \mathrm{O}$ during crystallisation disrupts the otherwise favourable window-to-window CC3- $R$ cage pairing, as found in $\mathbf{C C} 3 \alpha$, which has a cage-cage intermolecular interaction energy calculated to be around $150 \mathrm{~kJ} \mathrm{~mol}^{-1}$ (DFT-D3). ${ }^{7}$ Hence, the three cage windows pack in a relatively inefficient manner, forming three extrinsic cavities into which the methyl termini of the $\mathrm{Et}_{2} \mathrm{O}$ solvent molecules are directed (Fig. 2). The fourth cage window is occupied by the aromatic face of a neighbouring cage: this window-to-face interaction has a binding energy that was calculated to be $55 \mathrm{~kJ} \mathrm{~mol}^{-1}$ less favourable than the window-to-window interaction, ${ }^{7}$ but this is evidently compensated by favourable cage-solvent interactions.

Stacks of CC3- $R$ molecules are formed as a result of this window-to-face packing (Fig. 3). This packing is very similar to that reported previously for crystalline CC4- $R .^{12}$ For CC4- $R$, we observed a de-symmetrisation upon desolvation ( $R 3$ to $P 3)$ as a result of the CC4- $R$ molecules undergoing a screw-type rotation to afford what is referred to hereafter as $\mathbf{C C 4} \alpha$ (Fig. 1c). Similarly, desolvation of $(\mathrm{CC} 3-R) \cdot\left(\mathrm{Et}_{2} \mathrm{O}\right)_{3} \cdot \mathrm{CH}_{2} \mathrm{Cl}_{2}$ affords a new solvent-free, metastable polymorph of CC3-R, CC3 $\beta$. This is also accompanied by screw-type rotation of the cage molecules in the crystal lattice (Fig. 3; Section 1.3, Fig. S2-S11, ESI $\dagger$ ). Single-crystal-to-singlecrystal transformations have been reported for discrete host molecules such as metallocycles ${ }^{13}$ and also for extended frameworks, ${ }^{14}$ but preservation of single crystallinity is not typically observed when there is a substantial structural rearrangement. For CC3, loss of the guest solvent causes the cages to pack in a more frustrated arrangement in CC3 $\beta$, causing a significant contraction of the unit cell volume (5\%).
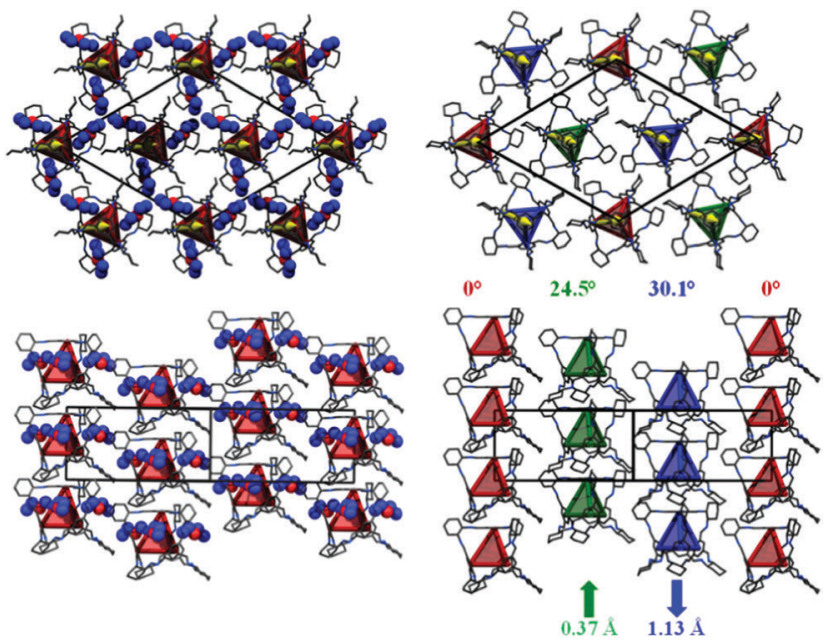

Fig. 3 Single crystal structure for $\mathrm{CC} 3-R \cdot\left(\mathrm{Et}_{2} \mathrm{O}\right)_{3} \cdot \mathrm{CH}_{2} \mathrm{Cl}_{2}$ collected at $100 \mathrm{~K}$ (left), viewed along [001] (above), along the direction of the cage stacks, and [110] (below), perpendicular to the cage stacks. $\mathrm{Et}_{2} \mathrm{O}$ solvent molecules shown as space filling representation, carbon atoms in blue. Intra-cavity $\mathrm{CH}_{2} \mathrm{Cl}_{2}$ solvent molecules and hydrogen atoms are omitted for clarity. Red tetrahedra represent the connectivity between four aromatic faces of the crystallographically equivalent CC3-R molecules. Single crystal structure for desolvated CC3 $\beta$ collected at $300 \mathrm{~K}$ (right). Red, green and blue tetrahedra represent the connectivity between the aromatic faces of the three crystallographically distinct CC3- $R$ molecules. Magnitude of rotation and translation of the cage molecules upon desolvation are indicated.

For $\mathbf{C C} 3 \beta$, the angle of cage rotation upon desolvation was defined as the angle rotated relative to the red cage (Fig. 3), which remains static and which, for convenience, has been located on the cell origin. For CC3, these rotation angles were determined to be $24.5^{\circ}$ and $30.1^{\circ}$, respectively, for the green and blue cages shown in Fig. 3. There is also a $0.37 \AA$ and $1.13 \AA$ shift, respectively, along the $z$-direction for the green and blue cages in a convergent manner. Unlike the thermodynamic desolvated polymorph $\mathbf{C C} 3 \alpha, \mathbf{C C} 3 \beta$ does not display any window-to-window packing of the cage molecules. $\mathbf{C C} 3 \alpha$ is predicted to be the lowest energy solvent-free form of CC3- $R,{ }^{8}$ and it is the most prevalent experimentally, being obtained from most solvents tested. CC3 $\alpha$ also exhibits the most efficient packing between cages and has the highest density. We suggest, therefore, that $\mathbf{C C} 3 \beta$ is a kinetically trapped polymorph, as supported by the occasional observation of the $\beta$-form when $\mathbf{C C} 3-R$ is rapidly precipitated from solvent by rotary evaporation.

Gas sorption analysis shows that $\mathbf{C C} 3 \beta$ is porous to nitrogen, with an apparent $\mathrm{SA}_{\mathrm{BET}}$ of $555 \mathrm{~m}^{2} \mathrm{~g}^{-1}$, and a microporous type I adsorption isotherm (Fig. 4). The nitrogen uptake and porosity for $\mathbf{C C} 3 \beta$ is higher than for $\mathbf{C C} 3 \alpha$, which can be rationalised by its lower density $\left(0.922 \mathrm{~g} \mathrm{~cm}^{-3}\right.$ for CC3 $\beta$ vs. $0.973 \mathrm{~g} \mathrm{~cm}^{-3}$ for $\left.\mathbf{C C} 3 \alpha\right)$. Hence, $\mathbf{C C} 3 \beta$ has extrinsic lattice sites that can accommodate additional $\mathrm{N}_{2}$ molecules. The $\mathbf{C C} 3 \beta$ polymorph is also porous to hydrogen and carbon dioxide, in both cases with a higher gas uptake than was found for the original $\mathbf{C C} 3 \alpha$ polymorph (Fig. S12 and S13, ESI $\dagger$ ). Even though the crystal packings for CC4 $\alpha$ and $\mathbf{C C} 3 \beta$ are essentially isostructural, the gas sorption isotherms are strikingly different. Gas-induced transformations 

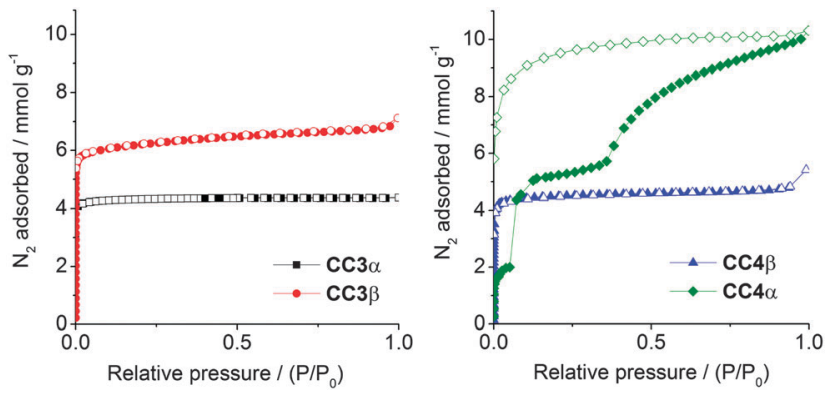

Fig. 4 Nitrogen sorption isotherms, at $77 k$, for $\mathrm{CC} 3 \alpha$ and $-\beta$ (left), and CC4 $\alpha$ and $-\beta$ (right). Adsorption isotherm branches are shown as solid symbols, and desorption branches as open symbols.

are known for organic solids. ${ }^{15}$ For $\mathbf{C C 4} \alpha$, there are low-pressure adsorption steps ${ }^{12}$ that are not observed for CC3 $\beta$. Also, CC4 $\alpha$ also adsorbs significantly more $\mathrm{N}_{2}$ than $\mathbf{C C} 3 \beta$ at higher relative pressures (Fig. 4). In situ powder XRD data indicates a sharp, low-pressure structural transition (Section 1.5, Fig. S14-S17, ESI $\dagger$ ). Analogous in situ powder XRD data for CC3 $\beta$ did not show any low-pressure transition (Fig. S18, ESI $\dagger$ ). The observation of a low-pressure structural transition for $\mathbf{C C 4} \alpha$, but not for CC3 $\beta$, might be due to the smaller cycloalkane vertex functionality in CC4. The more compact cyclopentyl vertices in CC4- $R$ might allow small rotational rearrangements of the cages at low $\mathrm{N}_{2}$ pressures, enabling the guest to gain more extensive access to the pore network at higher $\mathrm{N}_{2}$ pressures. By contrast, we speculate that the analogous rearrangements are hindered in CC3 $\beta$ by the bulkier cyclohexyl substituents, and a hence a classic type I isotherm is observed with no structural transition steps (Fig. 4).

Having directed CC3- $R$ to pack in the typical CC4- $R$ fashion (Fig. 1c), the reverse challenge was to direct CC4- $R$ to pack isostructurally with $\mathbf{C C} 3 \alpha$ (Fig. 1b). A recent computational study predicted a polymorph, $\mathbf{C C 4} \beta$, as the global lattice energy minimum for the homochiral CC4- $R .^{10}$ CC4 $\beta$ is isostructural with $\mathbf{C C} 3 \alpha$, suggesting initially that the same window-to-window cage pairing is also thermodynamically preferred for CC4- $R$. However, for CC4- $R$, the rigid-molecule constraint used in the CSP limits the reliability of the structure searches. Specifically, the experimental CC4 $\alpha$ structure involves close intermolecular contacts that distort the molecular geometry, and hence geometry-constrained CSP calculations lead to artificially high lattice energies for $\mathbf{C C 4} \alpha$. This was resolved by periodic DFT-D calculations, which showed the known $\mathbf{C C 4} \alpha$ polymorph to have a formation energy that is $8.19 \mathrm{~kJ} \mathrm{~mol}^{-1}$ more stable than $\mathbf{C C 4} \beta$ when molecular flexibility is taken into account. In the context of lattice energies, $8.19 \mathrm{~kJ} \mathrm{~mol}^{-1}$ is a relatively small difference, suggesting that CC4- $R$ might be directed to pack as CC4 $\beta$ by inclusion of an appropriate directing solvent.

A screen of 30 different crystallisation co-solvents was used in an attempt to access the $\mathbf{C C 4} \beta$ polymorph that was suggested by CSP (Section 1.6, Table S4, ESI $\dagger$ ). PXRD data showed that only one of the 30 co-solvents tested, para-xylene, produced a crystalline form that was distinct from $\mathbf{C C 4} \alpha$ (Fig. S19, ESI $\dagger$ ), again supporting the conclusion that $\mathbf{C C 4} \alpha$ is the thermodynamically most stable polymorph, as predicted computationally using DFT-D. ${ }^{10}$ We showed recently that para-xylene can occupy the interstitial site between two cage windows in the crystal lattice of CC3- $R,{ }^{16}$ and also that it can bridge adjacent windows in 1-D cage catenane chains. ${ }^{17}$ Here, para-xylene 'pegs' adjacent CC4- $R$ cage windows together in a similar fashion. Single crystals of this new CC4- $R$ phase were grown from a layered $\mathrm{CH}_{2} \mathrm{Cl}_{2}$-paraxylene solution (see Section 1.7, ESI $\dagger$ ). Single crystal X-ray diffraction revealed a new phase, CC4- $R \cdot\left(\mathrm{C}_{8} \mathrm{H}_{10}\right)_{3} \cdot\left(\mathrm{H}_{2} \mathrm{O}\right)_{2}$ had crystallised in the chiral orthorhombic space group $P 2_{1} 2_{1} 2_{1}$, where each of the four cage windows are penetrated by the methyl terminus of a para-xylene solvent molecule (Fig. 5a). The linear shape of the para-xylene molecule would therefore seem to be important, as observed for 1,4-dioxane in our previous study. ${ }^{9}$ This is supported by the fact that the structural isomers of this solvent, meta- and ortho-xylene (Table S4, ESI $\dagger$ ), did not produce the CC4 $\beta$ polymorph $(c f$., 1,3-dioxane in our previous work ${ }^{9}$ ).

In this structure, four para-xylene molecules are shared equally between two adjacent CC4- $R$ molecules. Extending this window pairing arrangement in three dimensions generates a diamondoid network filled with para-xylene solvent molecules (Fig. S20 and S21, ESI $\dagger$ ), reminiscent of the known CC3-R paraxylene solvate. ${ }^{16}$ However, unlike the CC3- $R$ solvate, one additional para-xylene solvent molecule per cage unit is located in extrinsic 1-D channels in the CC4- $R$ solvate (Fig. S21, ESI $\dagger$ ). As a result of this additional solvent molecule, desolvation to a symmetrical diamondoid pore network requires significant anisotropic contraction of the unit cell parameters, potentially resulting in a transformation to an alternate pore topology or loss of crystallinity. Remarkably, gradual heating of a solvate crystal of CC4- $R \cdot\left(\mathrm{C}_{8} \mathrm{H}_{10}\right)_{3} \cdot\left(\mathrm{H}_{2} \mathrm{O}\right)_{2}$ (Section 1.7 \& Fig. S22, ESI $\dagger$ ) resulted in preservation of single crystallinity and isolation of CC4 $\beta$ (Fig. $5 \mathrm{~b}$ and c), which was formed after a transformation of the crystal symmetry to the chiral cubic space group, $F 4_{1} 32$. This is structure is isostructural both with $\operatorname{CC} 3 \alpha$ and with the computationally predicted structure obtained for CC4 in CSP studies, as reported previously. ${ }^{10}$ This single-crystal-to-singlecrystal transformation was accompanied by a large contraction

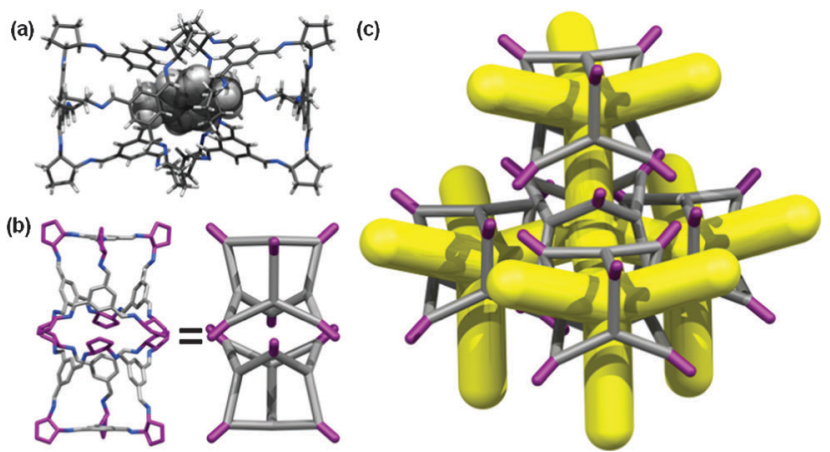

Fig. 5 (a) para-Xylene 'pegged' CC4- $R$ molecules from the single crystal structure CC4-R. $\left(\mathrm{C}_{8} \mathrm{H}_{10}\right)_{3} \cdot\left(\mathrm{H}_{2} \mathrm{O}\right)_{2}$; (b) direct window-to-window pairing arrangement displayed in CC4 $\beta$, generating (c) an interconnected diamondoid pore network upon desolvation of the xylene solvate. 
of the cell volume per CC4- $R$ molecule of around $13 \%$. The bulk CC4 $\beta$ material also remains crystalline after desolvation under heating and vacuum, and the resultant gas sorption properties very similar to those of $\mathbf{C C} 3 \alpha$, as expected from the isostructural packing. Indeed, the nitrogen sorption isotherms for CC3 $\alpha$ and CC4 $\beta$ are almost identical $\left(\mathrm{SA}_{\mathrm{BET}}\right.$ for $\mathrm{CC} 3 \alpha=409 \mathrm{~m}^{2} \mathrm{~g}^{-1} ; \mathrm{SA}_{\mathrm{BET}}$ for $\mathbf{C C 4} \beta=387 \mathrm{~m}^{2} \mathrm{~g}^{-1}$; Fig. 4). The preservation of single crystallinity upon desolvation is remarkable, given the large anisotropic contraction in the unit cell. The 'pegged' orientation of the CC4- $R$ molecules in the para-xylene solvate probably facilitates the preservation of this window-to-window packing after desolvation and prevents the cages from rearranging to form $\mathbf{C C 4} \alpha$. Transformation to $\mathbf{C C 4} \alpha$ would involve substantial and presumably high-energy reorganisation from the paraxylene solvate in order to generate the window-to-arene packing mode found in the thermodynamic polymorph.

Two organic cages, CC3- $R$ and $\mathbf{C C 4}-R$, were induced to interchange their low-energy packing modes by using directing solvents. This shows that polymorphism in porous molecular solids can to some extent be controlled by combining CSP calculations, intuitive design, and high-throughput crystallisation screens. We can identify specific co-solvents that either reinforce (para-xylene in $\mathbf{C C 4} \beta$ ) or disrupt $\left(\mathrm{Et}_{2} \mathrm{O}\right.$ in CC3 $\beta$ ) the solid-state window-to-window packing arrangement for these porous cages. Without the use of these directing co-solvents, the lowest energy $\alpha$-polymorph for CC3- $R$ is isostructural with the higher energy $\beta$-polymorph of CC4- $R$, and vice versa (Fig. 1b).

Isostructural crystal packings, however, can lead to quite different gas sorption properties: for example, CC4 $\alpha$ has marked steps in its isotherm, whereas CC3 $\beta$ does not. By contrast, the sorption isotherms for $\mathbf{C C} 3 \alpha$ and $\mathbf{C C 4} \beta$ are almost identical.

This represents a further step toward controlling the functional properties of porous molecular crystals by design. In the future, the a priori prediction of these solvent effects might also be possible, although at present this is prohibited by the computational expense of the relevant CSP methods.

We thank Diamond Light Source for access to beamline I11 (EE7040) that contributed to the results presented here and Prof. C. Tang, Dr P. Adamson, and Dr S. Thompson for their assistance during the experiment. We thank the EPSRC (EP/ H000925/1) and the ERC under FP7 (321156) for funding.

\section{Notes and references}

1 J. Tian, P. K. Thallapally and B. P. McGrail, CrystEngComm, 2012, 14, 1909-1919; M. Mastalerz, Angew. Chem., Int. Ed., 2010, 49, 5042-5053; L. J. Barbour, Chem. Commun., 2006, 1163-1168; J. L. Atwood, L. J. Barbour, A. Jerga and B. L. Schottel, Science, 2002, 298, 1000-1002; G. Zhang and M. Mastalerz, Chem. Soc. Rev., 2014, 43, 1934-1947.

2 C. S. Cundy and P. A. Cox, Chem. Rev., 2003, 103, 663-702.

3 O. M. Yaghi, H. Li, C. Davis, D. Richardson and T. L. Groy, Acc. Chem. Res., 1998, 31, 474-484; A. K. Cheetham, G. Férey and T. Loiseau, Angew. Chem., Int. Ed., 1999, 38, 3268-3292; S. Kitagawa, R. Kitaura and S.-i. Noro, Angew. Chem., Int. Ed., 2004, 43, 2334-2375; D. Bradshaw, J. B. Claridge, E. J. Cussen, T. J. Prior and M. J. Rosseinsky, Acc. Chem. Res., 2005, 38, 273-282.

4 A. P. Côté, A. I. Benin, N. W. Ockwig, M. O'Keeffe, A. J. Matzger and O. M. Yaghi, Science, 2005, 310, 1166-1170.

5 T. Tozawa, J. T. A. Jones, S. I. Swamy, S. Jiang, D. J. Adams, S. Shakespeare, R. Clowes, D. Bradshaw, T. Hasell, S. Y. Chong, C. Tang, S. Thompson, J. Parker, A. Trewin, J. Bacsa, A. M. Z. Slawin, A. Steiner and A. I. Cooper, Nat. Mater., 2009, 8, 973-978.

6 M. Mastalerz, M. W. Schneider, I. M. Oppel and O. Presly, Angew. Chem., Int. Ed., 2011, 50, 1046-1051; M. W. Schneider, I. M. Oppel, A. Griffin and M. Mastalerz, Angew. Chem., Int. Ed., 2013, 52, 3611-3615; A. Avellaneda, P. Valente, A. Burgun, J. D. Evans, A. W. Markwell-Heys, D. Rankine, D. J. Nielsen, M. R. Hill, C. J. Sumby and C. J. Doonan, Angew. Chem., Int. Ed., 2013, 52, 3746-3749.

7 T. Hasell, S. Y. Chong, K. E. Jelfs, D. J. Adams and A. I. Cooper, J. Am. Chem. Soc., 2012, 134, 588-598.

8 J. T. A. Jones, T. Hasell, X. Wu, J. Bacsa, K. E. Jelfs, M. Schmidtmann, S. Y. Chong, D. J. Adams, A. Trewin, F. Schiffman, F. Cora, B. Slater, A. Steiner, G. M. Day and A. I. Cooper, Nature, 2011, 474, 367-371.

9 T. Hasell, J. L. Culshaw, S. Y. Chong, M. Schmidtmann, M. A. Little, K. E. Jelfs, E. O. Pyzer-Knapp, H. Shepherd, D. J. Adams, G. M. Day and A. I. Cooper, J. Am. Chem. Soc., 2014, 136, 1438-1448.

10 E. O. Pyzer-Knapp, H. P. G. Thompson, F. Schiffmann, K. E. Jelfs, S. Y. Chong, M. A. Little, A. I. Cooper and G. M. Day, Chem. Sci., 2014, 5, 2235-2245.

11 A. J. Cruz-Cabeza, S. Karki, L. Fabian, T. Friscic, G. M. Day and W. Jones, Chem. Commun., 2010, 46, 2224-2226; A. J. Cruz-Cabeza, G. M. Day and W. Jones, Phys. Chem. Chem. Phys., 2011, 13, 12808-12816.

12 T. Mitra, X. Wu, R. Clowes, J. T. A. Jones, K. E. Jelfs, D. J. Adams, A. Trewin, J. Bacsa, A. Steiner and A. I. Cooper, Chem. - Eur. J., 2011, 17, 10235-10240.

13 L. Dobrzańska, G. O. Lloyd, H. G. Raubenheimer and L. J. Barbour, J. Am. Chem. Soc., 2005, 128, 698-699; G. O. Lloyd, H. G. Raubenheimer and L. J. Barbour, J. Am. Chem. Soc., 2005, 127, 13134-13135.

14 E. Y. Lee and M. P. Suh, Angew. Chem., Int. Ed., 2004, 43, 2798-2801; H. J. Choi and M. P. Suh, J. Am. Chem. Soc., 2004, 126, 15844-15851; S. K. Ghosh, W. Kaneko, D. Kiriya, M. Ohba and S. Kitagawa, Angew. Chem., Int. Ed., 2008, 47, 8843-8847.

15 P. K. Thallapally, B. Peter McGrail, S. J. Dalgarno, H. T. Schaef, J. Tian and J. L. Atwood, Nat. Mater., 2008, 7, 146-150.

16 T. Mitra, K. E. Jelfs, M. Schmidtmann, A. Ahmed, S. Y. Chong, D. J. Adams and A. I. Cooper, Nat. Chem., 2013, 5, 276-281.

17 T. Hasell, X. Wu, J. T. A. Jones, J. Bacsa, A. Steiner, T. Mitra, A. Trewin, D. J. Adams and A. I. Cooper, Nat. Chem., 2012, 2, 750-755. 